\title{
A major locus on chromosome 3 p22 conferring predisposition to human herpesvirus 8 infection
}

\author{
Vincent Pedergnana ${ }^{1,2}$, Antoine Gessain ${ }^{3}$, Patricia Tortevoye ${ }^{3}$, Minji Byun $^{4}$, Delphine Bacq-Daian ${ }^{5}$, \\ Anne Boland ${ }^{5}$, Jean-Laurent Casanova ${ }^{1,2,4}$, Laurent Abel ${ }^{\star, 1,2,4,6}$ and Sabine Plancoulaine ${ }^{\star, 1,2,3,6}$
}

\begin{abstract}
Infection with human herpesvirus 8 (HHV-8), the etiological agent of Kaposi's sarcoma, has been shown to display strong familial aggregation, in countries in which HHV-8 infection is endemic. We investigated 40 large families (608 subjects aged one to 88 years) living in an isolated area of Cameroon in which HHV-8 is highly endemic. We performed a two-step genetic analysis for HHV-8 infection status (HHV-8+/HHV-8- determined by immunofluorescence) consisting of an initial segregation analysis followed by a model-based genome-wide linkage analysis. Overall HHV-8 seroprevalence was $60 \%$, increasing with age. Segregation analysis provided strong evidence for a recessive major gene conferring predisposition to HHV-8 infection. This gene is predicted to have a major effect during childhood, with almost all homozygous predisposed subjects ( $\sim 7 \%$ of the population) becoming infected by the age of 10 . Linkage analysis was carried out on the 15 most informative families, corresponding to 205 genotyped subjects. A single region on chromosome 3 p22 was significantly linked to HHV-8 infection (LOD score=3.83, $P=2.0 \times 10^{-5}$ ). This study provides the first evidence that HHV-8 infection in children in endemic areas has a strong genetic basis involving at least one recessive major locus on chromosome 3 p22.
\end{abstract}

European Journal of Human Genetics (2012) 20, 690-695; doi:10.1038/ejhg.2011.260; published online 18 January 2012

Keywords: Kaposi's sarcoma-associated herpes virus; infection; linkage analysis; segregation analysis; endemic population

\section{INTRODUCTION}

Human herpesvirus 8 (HHV-8), first identified in 1994 in Kaposi's sarcoma (KS) skin lesions, ${ }^{1}$ is responsible for KS, primary effusion lymphoma and some forms of multicentric Castleman's disease. ${ }^{2,3}$ HHV-8 is transmitted principally through saliva, in which infectious viruses have been detected. ${ }^{4,5}$ In countries with low HHV-8 seroprevalence $(<5 \%)$ in the general population (eg, North America, Northern Europe, Asia), HHV-8 infection occurs mostly in homosexual men, and the main risk factors identified are HIV seropositivity, a large number of sexual partners and a history of sexually transmitted diseases $^{4,5}$ suggesting that HHV-8 transmission occurs during sex. In countries with high HHV-8 seroprevalence ( $>10 \%$; eg, Mediterranean Basin, Africa, South America), most individuals become infected during childhood and the main known risk factors are age and HIV coinfection. ${ }^{4,5}$ In these countries, several studies have also reported that the HHV-8 serological status of biological relatives is a risk factor for HHV-8 infection. Six studies have provided evidence of correlated HHV-8 serological status between mother and children, one in a Jewish population from Israel ${ }^{6}$ and five in the populations of African origin. $^{7-11}$ Three of these studies investigating dependences between other biological relatives also found that HHV-8 serological status was strongly correlated between siblings. ${ }^{8-10}$ This pattern of familial aggregation, which is not accounted for by known risk factors, suggests that host genetic factors may be involved in HHV-8 infection. This hypothesis was supported by a segregation analysis identifying a recessive gene controlling HHV-8 infection in the children of African origin living in French Guiana. ${ }^{12}$

However, few molecular human genetics studies have convincingly documented the genetic control of HHV-8-related phenotypes. One of the most remarkable findings to date is our recent discovery that classic KS in children has a Mendelian basis, in at least some children. We identified two children from the Mediterranean Basin with KS caused by an underlying primary immunodeficiency (PID): one with autosomal recessive complete IFN- $\gamma \mathrm{R} 1$ deficiency ${ }^{13}$ and the other with X-linked recessive Wiskott-Aldrich syndrome. ${ }^{14}$ Both the children had several additional clinical phenotypes. We then reported three unrelated Turkish children with classic KS in the absence of any other infectious or tumoral phenotype, each born to consanguineous parents, ${ }^{15}$ suggesting that isolated $\mathrm{KS}$ in children may also result from single-gene defects. Using the recently developed whole-exome sequencing approach in a Turkish child with early-onset fatal disseminated KS, we identified a homozygous splice-site mutation in STIM1 leading to complete STIM1 deficiency. ${ }^{16}$ As STIM1 deficiency is associated with a severe T-cell deficiency, ${ }^{17}$ this provided the first demonstration of a PID conferring a selective predisposition to isolated childhood KS upon HHV-8 infection. Less convincing results have been obtained for investigations of the role of common genetic variants in classic KS in adults, with only weak associations reported between polymorphisms of some immune-system-modulating genes (IL13, IL8RB and some HLA variants) and the risk of KS

\footnotetext{
${ }^{1}$ Laboratory of Human Genetics of Infectious Diseases, Necker Branch, Institut National de la Santé et de la Recherche Médicale U980, Paris, France; 2 Université Paris Descartes, Paris, France; ${ }^{3}$ Unité d'Epidémiologie et Physiopathologie des Virus Oncogènes, Institut Pasteur, Paris, France; ${ }^{4}$ St Giles Laboratory of Human Genetics of Infectious Diseases, The Rockefeller University, New York, NY, USA; ${ }^{5}$ Centre National de Génotypage, Evry, France

*Correspondence: Dr L Abel or S Plancoulaine, Laboratoire de Génétique Humaine des Maladies Infectieuses, Inserm U980, Faculté de Médecine Necker, 156 rue de Vaugirard, 75015 Paris, France. Tel: +331406156 89; Fax: +331406156 88; E-mail: laurent.abel@inserm.fr (LA) or sabine.plancoulaine@inserm.fr (SP)

${ }^{6}$ These authors contributed equally to this study.

Received 27 September 2011; revised 18 November 2011; accepted 7 December 2011; published online 18 January 2012
} 
development. ${ }^{18,19}$ Finally, one study investigated the role of 14 candidate genes in controlling the levels of antibodies directed against HHV-8 (ie, considered as a quantitative trait), and reported an association of some haplotypes encompassing the genes encoding IL-4, IL-6 and IL-12A with high anti-HHV-8 antibody titers. ${ }^{20}$ These association studies need to be replicated. By contrast, there has been no study of the molecular genetic basis of HHV-8 infection per se, defined as a binary phenotype (HHV-8+/HHV-8-). We report here the first genome-wide (GW) linkage scan searching for a major locus conferring predisposition to HHV-8 infection in a general population living in an area in which HHV-8 is endemic.

\section{MATERIALS AND METHODS}

\section{Study population}

The survey was carried out in a small isolated village located in the rainforest of the Ntem region of Southern Cameroon that has been described elsewhere. ${ }^{9}$ This work was carried out as a part of a multidisciplinary project (anthropological, medical, and economic) in which all the individuals living in this village were studied. All the subjects live in very similar conditions in dwellings close to each other, and have very homogeneous cultural and social behaviors, which allow assuming a similar level of exposure to HHV-8 for all study subjects. Information about family relationships was obtained from several interviews. Full pedigrees were constructed from this information, and were validated by the individuals concerned. All the subjects belonged to the Bantu ethnic group, and were mostly from the Fang tribe. Complete biological and epidemiologic data were available for 608 subjects, 287 of whom were male and 321 of whom were female. The study subjects were aged from 1 to 88 years (median 18 years) and clustered into 40 families, each of which had 3-51 members. This survey was carried out with the authorization of the national (Ministry of Health and the Cameroonian Ethics Committee) and local authorities (village chief), and information about the study was supplied to each participant. Approval was obtained from the institutional review board of the Institut Pasteur in Paris. Written informed consent was obtained from all the adult subjects and from the parents of minors.

\section{Biological methods}

Serological testing for HHV-8 was performed as previously described. ${ }^{9,21}$ This test which detects antibodies directed principally against lytic HHV-8 antigens is very suitable for use in epidemiologic studies and does not react with any other known HHV-8, including Epstein-Barr virus. It has been used by many groups leading to epidemiological results on HHV-8 that have been widely confirmed by different studies using the same or additional serological tests. Thus, we consider that a phenotype derived from this test is quite robust for epidemiological studies, and is fully appropriate for a genetic analysis. From this serological testing, we studied a binary phenotype: HHV-8-seropositive or -seronegative (HHV-8+/-) status. A sample was considered positive if cytoplasmic immunofluorescence was observed at a dilution of 1:20.

\section{Genotyping}

Genomic DNA was extracted from the blood samples. High-density genotyping for the linkage study was performed at the French National Genotyping Center (Evry, France), with the Infinium HumanLinkage-12 panel (Illumina, San Diego, CA, USA) containing 6090 single-nucleotide polymorphisms (SNPs). Forty-seven SNPs with a call rate $<80 \%$ were excluded. The mean call rate for the 6043 remaining SNPs was $99 \%$, and 5810 SNPs had a call rate $>99 \%$. Six hundred and seventy SNPs with a minor allele frequency $<5 \%$ were further excluded, as well as one of the 5373 remaining SNPs which showed departure from Hardy-Weinberg equilibrium among the founders at the 0.001 level. In total, 5372 high-quality SNPs were considered for the linkage analysis.

\section{Statistical methods}

Segregation analysis was carried out with a regressive logistic model, ${ }^{22}$ specifying a regression relationship between the probability of a person being infected (ie, to be HHV-8+) and a set of explanatory variables, including major gene, phenotype of preceding relatives and known risk factors. The use of this regressive model made it possible to analyze large families as a whole, to estimate genetic and risk factor effects simultaneously and to consider different patterns of familial correlation for HHV-8 status. ${ }^{9,10}$ The parameters of the major gene are the frequency of the allele conferring a predisposition to HHV-8 infection (D), and the three baseline risks of being HHV-8+ on the logit scale for the three genotypes (DD, Dd and dd), from which penetrance can be computed. Familial correlations were parameterized through regression coefficients accounting for the relationship between the phenotype of an individual and the phenotypes of his preceding relatives: father-mother, father-offspring, mother-offspring and sib-sib. Finally, the effects of covariates were specified through regression coefficients. Parameters were estimated by maximum likelihood, and nested models were compared by likelihood ratio tests. In particular, evidence for the involvement of a major gene is based on the rejection of a sporadic model including only familial correlations when compared with a genetic model including a major gene effect with the same pattern of familial correlations. When evidence of major gene involvement is found, then the additional tests are carried out to determine the mode of inheritance (dominant/recessive/additive) of that gene and to ensure that the familial transmission of this major gene is consistent with Mendelian expectations. ${ }^{12,23}$ Segregation analysis was performed with REGRESS software, ${ }^{24}$ which incorporates the regressive approach in the LINKAGE package..$^{25}$ All tests were carried out with a type I error of 0.05 . No ascertainment correction was required when calculating likelihood, because all the families of the village were included in the analysis.

We carried out the GW linkage analysis with the model generated from segregation analysis. According to our model, the penetrance of the allele conferring predisposition to HHV-8 infection was dependent on age (coded into three classes) and the HHV-8 status of preceding siblings, if any (three levels were considered: only HHV-8 - preceding siblings, at least one HHV-8+ preceding sibling, no preceding siblings). Thus, we had to include nine liability classes for each genotype in this analysis to account for all the combinations of factors influencing HHV-8 infection. Multipoint linkage analysis was performed on the most informative families of the studied population - those with a least one HHV-8+ child under the age of 10 years, with MERLIN software. ${ }^{26}$ A LOD score threshold of 3.3 was used to define significance $\left(P=4 \times 10^{-5}\right)$ as suggested in model-based linkage analysis. ${ }^{27}$

\section{RESULTS}

Evidence for a recessive major gene controlling HHV-8 infection This study was carried out in a population living in an isolated village located in the rainforest of the Ntem region of Southern Cameroon, which has been described elsewhere. ${ }^{9}$ The overall seroprevalence of HHV-8 was 59.9\% (364 HHV-8+ subjects among the 608 included) with no significant difference between male (59.9\%) and female $(59.8 \%)$ subjects. As previously observed, ${ }^{9,10}$ age had a strong effect on HHV-8 seroprevalence, which was $32 \%$ in subjects under the age of 9 years, increasing to about $62 \%$ between the ages of 10 and 39 years, and then to over $80 \%$ in older individuals ( $>39$ years; Figure 1). These three age classes $(1-9,10-39$ and $>39$ years old) gave the bestfitting coding scheme to account for the effect of age on HHV-8 seroprevalence and were therefore used for further analyses. Segregation analysis on the 40 families of this population provided significant evidence $(P=0.001)$ for a recessive major gene controlling HHV-8 infection, with residual sib-sib correlation. Additional tests showed that the familial transmission of this major gene was consistent with Mendelian expectations. Under this recessive gene model, the frequency of the predisposing allele, $\mathrm{D}$, was estimated at 0.27 , indicating that $7.3 \%$ of subjects were DD-homozygotes predisposed to HHV-8 infection.

The influence of age on penetrance - that is the probability of being HHV-8+ as a function of genotype - is shown for children under the age of 15 years in Figure 2a. By the age of 15 years, penetrance is predicted to be almost complete among DD predisposed children, reaching 0.89 for individuals with and 0.70 for individuals without a 


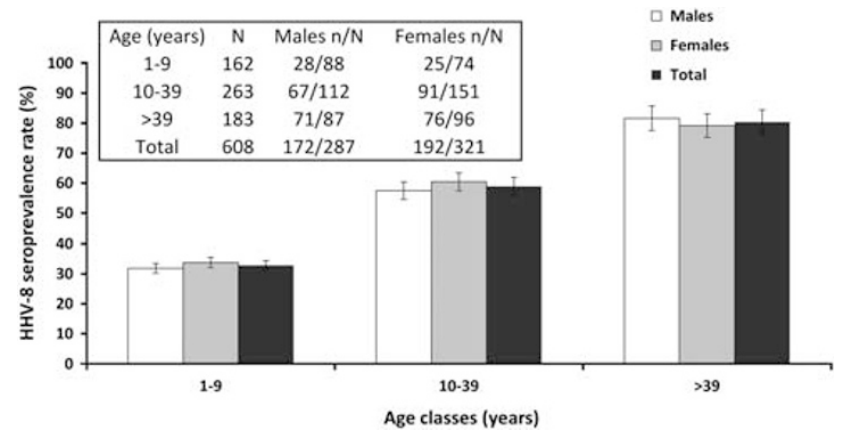

Figure 1 HHV-8 seroprevalence rates in 608 Bantu subjects from an isolated village in the Ntem Valley, South Cameroon, as a function of age, classified into three classes (1-9, 10-39 and >39 years old). Antibodies directed against lytic HHV-8 antigens were detected in immunofluorescence assays with the KS-1 cell line. Bars indicate the 95\% confidence intervals for seroprevalence rates.
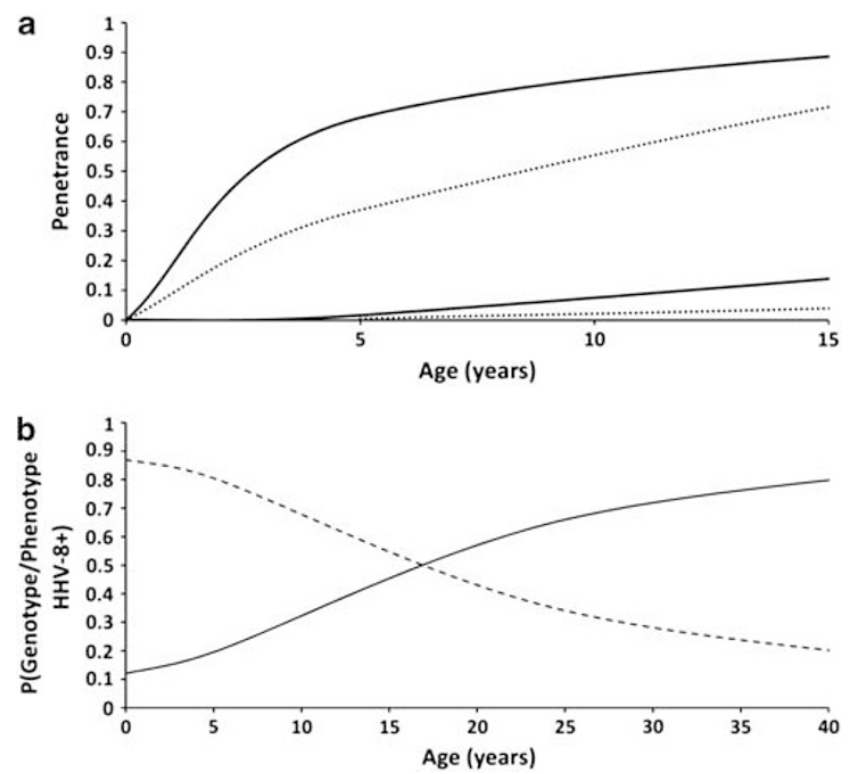

Figure 2 Characteristics of the recessive major gene conferring predisposition to HHV-8 infection, as predicted by the segregation analysis model. (a) Penetrance (ie, probability of being HHV-8 seropositive) for children under the age of 15 years, as a function of age, genotype for the detected major gene (DD, two upper lines; $\mathrm{Dd}$ or dd, two lower lines) and HHV-8 status of preceding siblings. $D$ is the recessive allele conferring predisposition to HHV-8 infection. Dashed lines and solid lines correspond to children without and with at least one HHV-8-seropositive sibling, respectively. (b) Variation with age of the probability of carrying either a predisposing genotype (DD, dashed line) or a non-predisposing genotype (Dd or dd, plain line), for HHV-8+ subjects.

HHV-8+ sibling, whereas it remained below 0.07 for Dd/dd nonpredisposed children with a HHV-8+ sibling. We then calculated the probability of carrying a given genotype (DD or Dd/dd) as a function of age in HHV-8+ subjects (Figure 2b). For HHV-8+ children, the probability of being DD (ie, having a predisposition to HHV-8 infection) was about 0.85 at the age of 2 years. This probability decreased with age, but was still $\sim 0.68$ at the age of 10 years and remained greater than the probability of being $\mathrm{Dd} / \mathrm{dd}$ until the age of 17 years. By contrast, the probability of being Dd/dd in HHV-8+ adults was much greater than the probability of being DD (eg, $0.85 \mathrm{vs}$
0.15 at the age of 40$)$. This finding is entirely consistent with the overall seroprevalence of HHV-8 in the adult population $(\sim 60 \%)$ and the much lower overall frequency of DD predisposed subjects $(\sim 7 \%)$. These data clearly indicate that most HHV-8+ adults correspond to sporadic (ie, Dd/dd genotype) cases of infection, whereas most HHV$8+$ subjects under the age of 10 years are genetic (ie, DD genotype) cases.

\section{Mapping of the major gene to chromosome 3 p22}

For the mapping of the recessive major gene locus identified in the previous segregation analysis, we carried out a GW linkage study on the most informative families (ie, the families most likely to contain genetic cases). We selected the families with at least one HHV-8+ child under the age of 10 years, as the probability of being a genetic case is greater than twice the probability of being a sporadic case for HHV-8+ children $<10$ years of age (Figure $2 b$ and previous paragraph). Fifteen families met these criteria (Supplementary Figure). These families included 205 subjects - 103 female and 102 male subjects - who were genotyped for the SNPs of the Illumina HumanLinkage-12 panel. Linkage information content (IC) was very high across all autosomes, with a mean GW information level of $90 \%$ (from 76 to $96 \%$ ). The model-based GW linkage analysis provided a single peak with significant evidence for linkage on chromosome $3 \mathrm{p} 22$ with a LOD score of $3.83\left(P=2.0 \times 10^{-5}, \mathrm{IC}=95 \%\right.$; Figure $\left.3 \mathrm{a}\right)$. The maximum multipoint LOD score was obtained for marker rs1455326, corresponding to a physical position of $35.42 \mathrm{Mb}$ on chromosome 3 . The $95 \%$ confidence interval (corresponding to a 1.5 LOD decrease from the chromosomal position with the peak LOD $\operatorname{score}^{28}$ ) defined a 5.35-Mb chromosomal distance (from SNP rs4796 to SNP rs2226462). This region contains 36 genes (17 of known function) and two microRNA sequences (Figure $3 b$ ).

Only seven large multigenerational families contributed positively to this peak, the eight remaining families providing slightly negative LOD scores (Supplementary Figure). These seven families contained a total of 138 individuals (family size of 6-51 members), including 44 genotyped children under the age of 10 years, 25 of whom were HHV-8+ (1-5 HHV-8+ children under the age of 10 years per family) and 19 of whom were HHV-8-. Although age was taken into account in the analysis, it was possible that some HHV-8 - children could be misclassified as they may have not been exposed yet and may become infected later once exposed. To assess the influence of HHV-8individuals in the analysis, we also conducted a robust linkage analysis considering only HHV-8+ subjects (similar to a classical affected-only analysis). We found again a linkage peak at rs1455326 with a reduced LOD score at $1.9(P=0.003)$ showing that removing HHV-8- subjects led to a decrease in the LOD score from 3.8 to 1.9. This observation clearly indicates that the large majority of these HHV-8- subjects brought additional linkage information to the $3 \mathrm{p} 22$ region, and could not be misclassified (in particular, phenotype misclassification is known to decrease the power of linkage analysis). Finally, we conducted a linkage analysis restricted to the eight families displaying no linkage to chromosome $3 \mathrm{p} 22$. This analysis generated no global LOD score $>1.2$ arguing against the presence of a second major locus that would be common to most of these families, and rather suggesting genetic heterogeneity involving several different loci.

\section{DISCUSSION}

Here, we report the first GW linkage study in a general population in which HHV-8 is highly endemic (overall seroprevalence of $60 \%$ ) to map a major locus conferring predisposition to HHV-8 infection. The linkage analysis was based on the model generated by segregation 

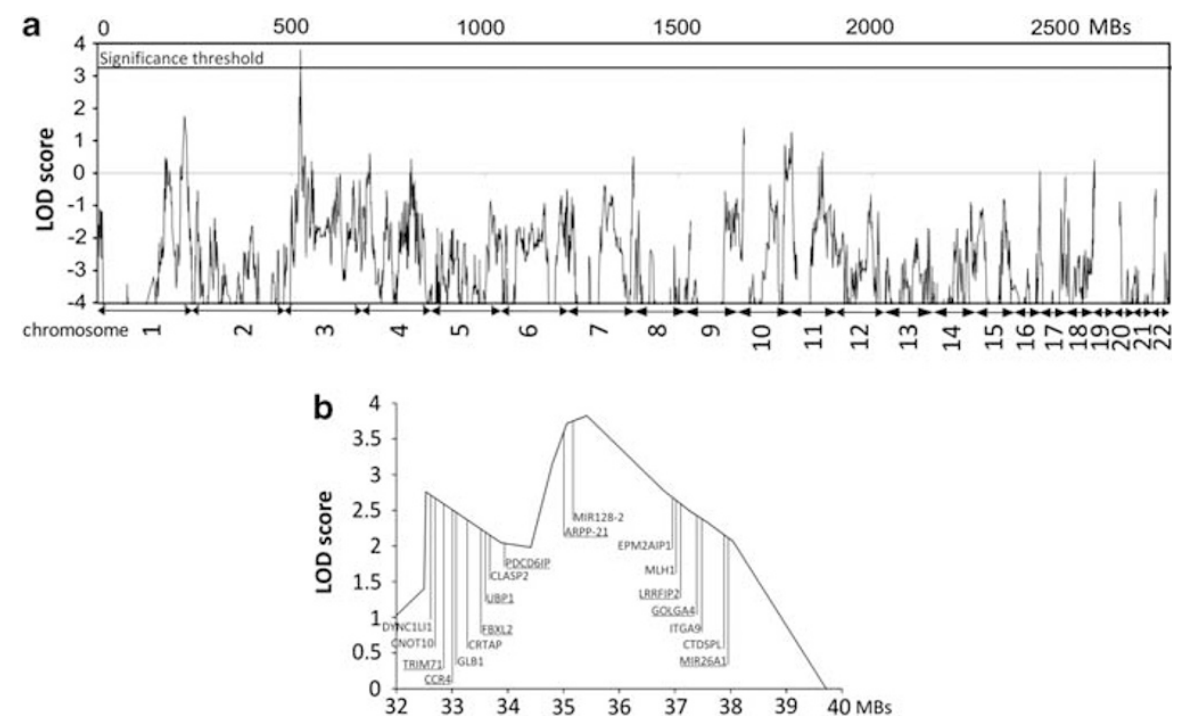

Figure 3 Genome-wide linkage analysis in 15 Cameroonian families with at least one HHV-8+ child under the age of 10 years. (a) Multipoint LOD scores ( $y$ axis) are plotted along the 22 autosomes (lower $x$ axis: chromosome numbers, upper $x$ axis: physical distances in megabases). (b) Expanded view of the region with the highest LOD score on chromosome 3p22. Genes with known functions and microRNA sequences located within the $95 \%$ confidence interval of the peak are indicated, and those involved in antiviral response and immunity are underlined.

analysis, which provided clear evidence for a recessive major gene conferring a strong predisposition to HHV-8 infection in children under the age of 10 years. According to this recessive model, about $7 \%$ of the population is homozygous for the $\mathrm{D}$ allele conferring predisposition to HHV-8 infection. These results are entirely consistent with the results we obtained in a previous segregation analysis on a general population from French Guiana with a lower seroprevalence of HHV8 (overall seroprevalence of $12 \%$ ). ${ }^{12}$ In both the populations, we found evidence for the involvement of a recessive gene in susceptibility to HHV-8 infection, with $\sim 7 \%$ of the population homozygous for the allele conferring predisposition to HHV-8 infection, and a major effect in childhood. Both these populations are of African origin, but HHV8 -seroprevalence was much higher in Cameroon, suggesting the existence of additional risk factors of a genetic and/or environmental nature (related to behavioral, cultural, or social conditions), which remain to be investigated and identified.

The major gene concerned is principally responsible for HHV-8 infection in children, and most HHV-8 infections in adults are not explained by this locus. These observations are consistent with our general hypothesis of an age-dependent genetic architecture of infectious diseases. ${ }^{29}$ According to this hypothesis, genetic predisposition to infectious diseases in children, particularly during primary infection, results principally from single-gene variations of variable clinical penetrance, whereas predisposition to infectious diseases is more complex in adults. The findings reported here suggest that this model could be extended from infectious diseases to infection, with infection in childhood being mostly controlled by major genes. Predisposition to infection in adults, which may be related to repeated exposure, would also be expected to be more complex and should involve additional risk factors, such as polygenic variation, age-dependent somatic and epigenetic factors associated with impaired immunity, and/or a cumulative effect of viral exposure and ecological variation. Even during childhood, the major gene detected in this study cannot account for all cases of predisposition as some families were not linked to this locus. This suggests the existence of genetic heterogeneity, which is also consistent with several observations in the human genetics of infectious diseases, including (1) the demonstrated role of a collection of rare variants (in different genes) in the most severe infectious diseases in childhood, including disseminated mycobacterial infections, invasive pneumococcal diseases and herpes simplex encephalitis; ${ }^{29}$ (2) the identification, by positional cloning, of two major loci having a major role in early-onset forms of leprosy - PARK2/PACRG variants, ${ }^{30}$ and $L T A$ variants ${ }^{31}$ - with additional genetic effects yet to be identified; (3) the evidence, provided by linkage analysis, that there are two major loci controlling tuberculosis infection, as assessed by the tuberculin skin test. ${ }^{32}$

The model-based GW linkage analysis in Cameroon identified a unique region of interest that mapped to chromosome $3 \mathrm{p} 22$, with a significant LOD score of 3.83. This region contains eight genes and one microRNA sequence that appeared potentially more interesting than the others, because they are thought to be involved in antiviral immunity or immune responses (genes and microRNA sequence underlined in Figure 3b). Three of these genes have been reported to be involved in the replication of both HIV (PDCD6IP, UBP $P^{33,34}$ ) and HCV $\left(F B X L 2^{35}\right)$ in vitro. An isoform, denoted as TARPP, of the protein encoded by ARPP-21 located close to the linkage peak is involved in the regulation of early T-cells development. ${ }^{36,37}$ Another gene (LRRFIP2) encodes a leucine-rich repeat protein interacting with MyD88 and modulating Toll-like receptor signaling. ${ }^{38}$ The CCR4 gene encodes the receptor of two chemokines, TARC/CCL17 and MDC/CCL22, that are produced by dendritic cells. These molecules regulate trafficking of leukocytes such as macrophages and monocytes for MDC and T lymphocytes (in particular Treg cells) for TARC. ${ }^{39,40}$ Interestingly, the HHV-8-encoded chemokine vMIP-III was shown to be an agonist of CCR4 and to preferentially chemoattract Th2 cells. ${ }^{41,42}$ Finally, miR26a was shown to be involved in IFN- $\beta$ production in human cells. ${ }^{43}$

GW linkage analysis is a powerful strategy for identifying major loci underlying infections or infectious diseases. It has already been successfully applied to various populations and has resulted in the mapping of major loci involved in schistosomiasis, ${ }^{44,45}$ leprosy, ${ }^{46,47}$ tuberculosis $^{32,48}$ and HTLV-1 infection. ${ }^{49}$ The next step in this 
positional cloning strategy is linkage disequilibrium (LD) mapping through association studies, using a dense map of polymorphisms. Following this strategy, which has already proved successful in studies of leprosy, ${ }^{30,31} \mathrm{LD}$ mapping will be conducted in the 3 p22 region, with an independent sample of the same genetic background as our original Cameroonian sample, to search for the genetic variants of the major locus conferring predisposition to HHV-8 infection in childhood. The identification of such variants should greatly improve our understanding of the molecular mechanisms involved in the response to HHV-8 infection and may also unravel new pathways for investigation in HHV-8-associated diseases, such as KS and multicentric Castleman's disease.

\section{CONFLICT OF INTEREST}

The authors declare no conflict of interest.

\section{ACKNOWLEDGEMENTS}

We thank the families from the Ntem valley for their participation in this study; Alain Froment, Philippe Tuppin, Edouard Betsem and Richard Njouom for assistance with data collection; and all members of the two branches of the Laboratory of Human Genetics of Infectious Diseases for fruitful discussions. This work was supported by INSERM, Institut Pasteur and Centre National de Génotypage, Centre National de la Recherche Scientifique (CNRS, Comité Environnement et Santé), Association pour la Recherche contre le Cancer (ARC), Programme National de Recherche Fondamentale en Microbiologie et Maladies Infectieuses et Parasitaires, Institut Pasteur (action concertee Inter-Pasteurienne for 'HHV-8 and Kaposi's Sarcoma in intertropical countries'), the Rockefeller University Center for Clinical and Translational Science grant number 5UL1RR024143, The Rockefeller University and St Giles Foundation. SP received funding from Assistance-Publique-Hôpitaux de Paris. MB was supported by the Irvington Institute Fellowship Program of the Cancer Research Institute.

1 Chang Y, Cesarman E, Pessin MS et al: Identification of herpesvirus-like DNA sequences in AIDS-associated Kaposi's sarcoma. Science 1994; 266: 1865-1869.

2 Gessain A, Briere J, Angelin-Duclos C et al: Human herpes virus 8 (Kaposi's sarcoma herpes virus) and malignant lymphoproliferations in France: a molecular study of 250 cases including two AIDS-associated body cavity based lymphomas. Leukemia 1997; 11: 266-272.

3 Gessain A, Sudaka A, Briere J et al: Kaposi sarcoma-associated herpes-like virus (human herpesvirus type 8) DNA sequences in multicentric Castleman's disease: is there any relevant association in non-human immunodeficiency virus-infected patients? Blood 1996; 87: 414-416.

4 Pica F, Volpi A: Transmission of human herpesvirus 8: an update. Curr Opin Infect Dis 2007; 20: 152-156.

5 Uldrick TS, Whitby D: Update on KSHV epidemiology, Kaposi Sarcoma pathogenesis, and treatment of Kaposi Sarcoma. Cancer Lett 2011; 305: 150-162.

6 Davidovici B, Karakis I, Bourboulia D et al: Seroepidemiology and molecular epidemiology of Kaposi's sarcoma-associated herpesvirus among Jewish population groups in Israel. J Natl Cancer Inst 2001; 93: 194-202.

7 Malope BI, Pfeiffer RM, Mbisa G et al: Transmission of Kaposi sarcoma-associated herpesvirus between mothers and children in a South African population. J Acquir Immune Defic Syndr 2007; 44: 351-355.

8 Mbulaiteye SM, Pfeiffer RM, Whitby D, Brubaker GR, Shao J, Biggar RJ: Human herpesvirus 8 infection within families in rural Tanzania. J Infect Dis 2003; 187: 1780-1785.

9 Plancoulaine S, Abel L, Tregouet D et al: Respective roles of serological status and blood specific antihuman herpesvirus 8 antibody levels in human herpesvirus 8 intrafamilial transmission in a highly endemic area. Cancer Res 2004; 64: 8782-8787.

10 Plancoulaine $\mathrm{S}$, Abel L, van Beveren $\mathrm{M}$ et al: Human herpesvirus 8 transmission from mother to child and between siblings in an endemic population. Lancet 2000; 356: 1062-1065.

11 Bourboulia D, Whitby D, Boshoff $C$ et al: Serologic evidence for mother-to-child transmission of Kaposi sarcoma-associated herpesvirus infection. JAMA 1998; 280: 31-32.

12 Plancoulaine S, Gessain A, van Beveren M, Tortevoye P, Abel L: Evidence for a recessive major gene predisposing to human herpesvirus 8 (HHV-8) infection in a population in which HHV-8 is endemic. J Infect Dis 2003; 187: 1944-1950.
13 Camcioglu Y, Picard C, Lacoste V et al: HHV-8-associated Kaposi sarcoma in a child with IFNgammaR1 deficiency. J Pediatr 2004; 144: 519-523.

14 Picard C, Mellouli F, Duprez R et al: Kaposi's sarcoma in a child with Wiskott-Aldrich syndrome. Eur J Pediatr 2006; 165: 453-457.

15 Sahin G, Palanduz A, Aydogan G et al: Classic Kaposi sarcoma in 3 unrelated Turkish children born to consanguineous kindreds. Pediatrics 2010; 125: e704-e708.

16 Byun M, Abhyankar A, Lelarge $\mathrm{V}$ et al: Whole-exome sequencing-based discovery of STIM1 deficiency in a child with fatal classic Kaposi sarcoma. J Exp Med 2010; 207: 2307-2312.

17 Picard C, McCarl CA, Papolos A et al: STIM1 mutation associated with a syndrome of immunodeficiency and autoimmunity. N Engl J Med 2009; 360: 1971-1980.

18 Brown EE, Fallin D, Ruczinski I et al: Associations of classic Kaposi sarcoma with common variants in genes that modulate host immunity. Cancer Epidemiol Biomarkers Prev 2006; 15: 926-934.

19 Masala MV, Carcassi C, Cottoni F, Mulargia M, Contu L, Cerimele D: Classic Kaposi's sarcoma in Sardinia: HLA positive and negative associations. Int J Dermato/ 2005; 44: 743-745.

20 Brown EE, Fallin MD, Goedert JJ et al: Host immunogenetics and control of human herpesvirus-8 infection. J Infect Dis 2006; 193: 1054-1062.

21 Plancoulaine S, Abel L, van Beveren M, Gessain A: High titers of anti-human herpesvirus 8 antibodies in elderly males in an endemic population. $J$ Natl Cancer Inst 2002; 94: 1333-1335.

22 Bonney GE: Regressive logistic models for familial disease and other binary traits. Biometrics 1986; 42: 611-625.

23 Plancoulaine S, Gessain A, Joubert M et al: Detection of a major gene predisposing to human T lymphotropic virus type I infection in children among an endemic population of African origin. J Infect Dis 2000; 182: 405-412.

24 Demenais F, Lathrop GM: REGRESS: A computer program including the regressive approach into the LINKAGE programs. Genet Epidemiol 1994; 11: 291.

25 Lathrop GM, Lalouel JM, Julier C, Ott J: Strategies for multilocus linkage analysis in humans. Proc Natl Acad Sci USA 1984; 81: 3443-3446.

26 Abecasis GR, Cherny SS, Cookson WO, Cardon LR: Merlin - rapid analysis of dense genetic maps using sparse gene flow trees. Nat Genet 2002; 30: 97-101.

27 Lander E, Kruglyak L: Genetic dissection of complex traits: guidelines for interpreting and reporting linkage results. Nat Genet 1995; 11: 241-247.

28 Dupuis J, Siegmund D: Statistical methods for mapping quantitative trait loci from a dense set of markers. Genetics 1999; 151: 373-386.

29 Alcais A, Quintana-Murci L, Thaler DS, Schurr E, Abel L, Casanova JL: Life-threatening infectious diseases of childhood: single-gene inborn errors of immunity? Ann NY Acad Sci 2010; 1214: 18-33.

30 Mira MT, Alcais A, Nguyen VT et al: Susceptibility to leprosy is associated with PARK2 and PACRG. Nature 2004; 427: 636-640.

31 Alcais A, Alter A, Antoni G et al: Stepwise replication identifies a low-producing lymphotoxin-alpha allele as a major risk factor for early-onset leprosy. Nat Genet 2007; 39: 517-522.

32 Cobat A, Gallant CJ, Simkin L et al: Two loci control tuberculin skin test reactivity in an area hyperendemic for tuberculosis. J Exp Med 2009; 206: 2583-2591.

33 Fujii K, Munshi UM, Ablan SD et al: Functional role of Alix in HIV-1 replication. Virology 2009; 391: 284-292.

34 Kato H, Horikoshi M, Roeder RG: Repression of HIV-1 transcription by a cellular protein. Science 1991; 251: 1476-1479.

35 Wang C, Gale Jr M, Keller BC et al: Identification of FBL2 as a geranylgeranylated cellular protein required for hepatitis C virus RNA replication. Mol Cell 2005; 18: 425-434.

36 Kisielow J, Nairn AC, Karjalainen K: TARPP, a novel protein that accompanies TCR gene rearrangement and thymocyte education. Eur J Immunol 2001; 31: 1141-1149.

$37 \mathrm{Kim} \mathrm{J}$, Lee J, Yadav $\mathrm{N}$ et al: Loss of CARM1 results in hypomethylation of thymocyte cyclic AMP-regulated phosphoprotein and deregulated early $\mathrm{T}$ cell development. J Biol Chem 2004; 279: 25339-25344.

38 Dai P, Jeong SY, Yu Y et al: Modulation of TLR signaling by multiple MyD88-interacting partners including leucine-rich repeat Fli-I-interacting proteins. J Immuno/ 2009; 182: 3450-3460.

39 Iellem A, Mariani M, Lang R et al: Unique chemotactic response profile and specific expression of chemokine receptors CCR 4 and CCR8 by CD4(+)CD25(+) regulatory T cells. J Exp Med 2001; 194: 847-853.

40 Andrew DP, Ruffing N, Kim CH et al: C-C chemokine receptor 4 expression defines a major subset of circulating nonintestinal memory T cells of both Th1 and Th2 potential. J Immunol 2001; 166: 103-111.

41 Stine JT, Wood C, Hill M et al: KSHV-encoded CC chemokine VMIP-III is a CCR4 agonist, stimulates angiogenesis, and selectively chemoattracts $\mathrm{TH} 2$ cells. Blood 2000; 95: 1151-1157.

42 Moore PS, Boshoff C, Weiss RA, Chang Y: Molecular mimicry of human cytokine and cytokine response pathway genes by KSHV. Science 1996; 274: 1739-1744.

43 Witwer KW, Sisk JM, Gama L, Clements JE: MicroRNA regulation of IFN-beta protein expression: rapid and sensitive modulation of the innate immune response. $J$ Immunol 2010; 184: 2369-2376.

44 Dessein AJ, Hillaire D, Elwali NE et al: Severe hepatic fibrosis in Schistosoma mansoni infection is controlled by a major locus that is closely linked to the interferon-gamma receptor gene. Am J Hum Genet 1999; 65: 709-721.

45 Marquet S, Abel L, Hillaire D et al: Genetic localization of a locus controlling the intensity of infection by Schistosoma mansoni on chromosome 5q31-q33. Nat Genet 1996; 14: 181-184. 
46 Mira MT, Alcais A, Van Thuc $N$ et al: Chromosome 6q25 is linked to susceptibility to leprosy in a Vietnamese population. Nat Genet 2003; 33: 412-415.

47 Siddiqui MR, Meisner S, Tosh $\mathrm{K}$ et al: A major susceptibility locus for leprosy in India maps to chromosome 10p13. Nat Genet 2001; 27: 439-441.
48 Baghdadi JE, Orlova M, Alter A et al: An autosomal dominant major gene confers predisposition to pulmonary tuberculosis in adults. J Exp Med 2006; 203: 1679-1684.

49 Plancoulaine S, Gessain A, Tortevoye P et al: A major susceptibility locus for HTLV-1 infection in childhood maps to chromosome 6q27. Hum Mol Genet 2006; 15: 3306-3312.

Supplementary Information accompanies the paper on European Journal of Human Genetics website (http://www.nature.com/ejhg) 